\title{
DYNAMIC TESTING OF FULL-SCALE NUCLEAR POWER PLANT STRUCTURES AND EQUIPMENT
}

\author{
C.B. SMITH \\ Applied Nucleonics Company, Inc., Los Angeles, California 90024, U.S.A.
}

\section{SUMMARY}

Three steps are required in the design of reliable nuclear power plants to be located in seismic areas. In addition to development of realistic analytical models, it is necessary to perform dynamic tests to verify the models and acquire information needed to establish the dynamic parameters for modeling. The third and final step is to perform high level proof tests to validate analysis and test results. This report is an overview of dynamic testing methods.

Testing can be performed in the laboratory or in the field. Laboratory tests are useful because a wide range of effects can be studied and test parameters are more easily controlled. Care must be exercised to insure that the laboratory situation faithfully reproduces the actual structure in such details as supports, appurtenances, appendages, and mounting methods. In general, laboratory methods permit high level excitation of structures weighing up to a few metric tons (a few facilities in the world have capabilities up to 100 metric tons).

Since actual structures of interest to nuclear power plant designers often weigh up to ten thousand metric tons, field test- 
ing is also important. Test procedures have been developed, using portable structural vibrators, for testing structures as large as nuclear power plant containment buildings. High level tests can be performed using explosives buried in the soil to excite structures. Recent work by the author demonstrates that explosive tests can be conducted which produce a predetermined, specified response spectrum (see Paper $K 2 / 10)$.

An important feature of field testing is the fact that earthquake effects are most closely simulated. Effects such as those due to local soils, the interaction of the soil and foundations, and field installation procedures are automatically taken into account.

In conclusion, the practicing earthquake engineer should be aware that practical methods exist for testing virtually any type of structure anywhere in the world. While it is, of course, impossible to "test" a non-existont first-of-a-kind structure, it is often possible to utilize similar existing structures or models to obtain test data. When equipment or structures are available, testing should be used to supplement analysis. Surprisingly, the cost of testing complex structures is often much less than analysis. Based on actual tests, costs have been about $0.01 \%$ of the cost of the structure for nuclear power plants.

The advantages of proof-testing to insure confidence in design and to facilitate licensing are obvious. Less obvious, perhaps, is the fact that test data can permit design economies and cost savings by eliminating overdesign or unnecessary conservatism. 